\title{
QUANTUM BACKREACTION (CASIMIR) EFFECT WITHOUT INFINITIES: ALGEBRAIC ANALYSIS
}

\author{
ANDRZEJ HERDEGEN \\ Institute of Physics, Jagiellonian University, \\ Reymonta 4, 30-059 Kraków, Poland \\ herdegen@th.if.uj.edu.pl
}

Published 28 July 2012

\begin{abstract}
Casimir effect, in most general terms, is the backreaction of a quantum system responding to an adiabatic change of external conditions. This backreaction is expected to be quantitatively measured by a change in the expectation value of a certain energy observable of the system. However, for this concept to be applicable, the system has to retain its identity in the process. Most prevailing tendencies in the analysis of the effect seem to ignore this question.

In general, a quantum theory is defined by an algebra of observables, whose representations by operators in a Hilbert space define concrete physical systems described by the theory. A quantum system retains its identity if both the algebra as well as its representation do not change. We discuss the resulting restrictions for admissible models of changing external conditions. These ideas are applied to quantum field models. No infinities arise, if the algebraic demands are respected.
\end{abstract}

PACS numbers: 03.70.+k, 03.65.Bz, 11.10.-z

\section{Introduction}

Quantum theory, as anticipated already by Dirac, is most appropriately developed in algebraic terms. This view found later strong confirmation when it turned out that canonical commutation relations for systems with infinite number of degrees of freedom have infinite number of inequivalent representations by operators in a Hilbert space. This lead Haag and Kastler in 1955 to the formulation of the algebraic framework for the quantum field theory, which turned over the following decades to be the most appropriate basis for investigation of fundamental properties of quantum theory (see Ref. 1).

In this contribution I give a very brief sketch of the analysis of Casimir effect from the algebraic point of view developed in a series of papers, Refs. 2-5. Theoretical description of this effect turns out to be crucially dependent on algebraic aspects of quantum fields. For models respecting consistency conditions resulting from this analysis no infinities arise. For detailed exposition and discussion we refer the reader to the cited articles.

\section{Quantum system under external conditions}

\section{System Q-M}

- $\mathrm{Q}$ - a relatively simple quantum system (e.g. a quantum field), 
- $\mathrm{M}$ - a complex macroscopic system (say, conducting plates) with collective effective coordinates $a$.

- Full closed theory of Q-M out of reach.

- Approximation: $\mathrm{M}$ is 'heavy' - characterized by very large inertia; thus:

- variables $a$ are classical,

- changes of $a$ are adiabatic.

\section{Isolated system $Q$ (Heisenberg picture)}

- Basic quantum variables at a fixed time form an abstract algebra $\mathcal{A}$, e.g. a CCR algebra.

- Algebra is represented by operators in a Hilbert space $\mathcal{H}$ :

$$
\pi: \mathcal{A} \mapsto \pi(\mathcal{A}), \quad A \mapsto \pi(A) .
$$

Density operators in $\mathcal{H}$ represent states of the system $Q$.

- Intrinsic dynamics of $Q$ defined by an automorphism of $\mathcal{A}$ :

$$
\alpha_{t}: \mathcal{A} \mapsto \mathcal{A}, \quad A \mapsto \alpha_{t} A
$$

implemented by a unitary evolution in the Hilbert space $\mathcal{H}$ :

$$
\pi\left(\alpha_{t} A\right)=U(t) \pi(A) U(t)^{*}, \quad U(t)=\exp (i t H),
$$

where $H$ - the energy operator of the system, with nonnegative spectrum and a ground state, represented by a unit eigenvector; energy may be normalized to be zero in that state.

\section{Introducing $M$}

- Add part $M$ into the system: characterized by classical variables $a$; no quantum degrees added.

- System $Q$ should retain its identity: algebra $\mathcal{A}$ must remain unaffected (unchanged).

- States to be considered must be physically comparable: the representation $\pi$ of $\mathcal{A}$ must remain unaffected.

\section{Dynamics of $Q$ with frozen $M$}

- Degrees a frozen - system Q still a closed system in interaction with conditions created by $M$; for each $a$ evolution: an automorphism of $\mathcal{A}$ :

$$
\alpha_{a t}: \mathcal{A} \mapsto \mathcal{A}, \quad A \mapsto \alpha_{a t} A .
$$

- Evolution implemented in the representation $\pi$ : for each $a$

$$
\pi\left(\alpha_{a t} A\right)=U_{a}(t) \pi(A) U_{a}(t)^{*}, \quad U_{a}(t)=\exp \left(i t H_{a}\right) .
$$

- For each $a$ the generator $H_{a}$ defined by this up to:

$$
H_{a} \rightarrow H_{a}+\lambda_{a} \text { id }
$$

where $\lambda_{a}$ is any real function of parameters $a$. 


\section{Coupled system Q-M (Schrödinger picture)}

- Unitary evolution of $Q$ in Schrödinger picture (Q not closed - evolution on algebraic level: too restrictive).

- Suppose that $a(t)$ is known as a 'slow' function of time (system $M$ is 'heavy'; 'slow' - with respect to the time-scale determined by the evolution with fixed $a$ ).

Adiabatic approximation with initial $(t=0)$ eigenstate of $H_{a(0)}$ :

$$
\psi(t)=e^{i \varphi(t)} \psi_{a(t)},
$$

where $H_{a} \psi_{a}=E_{a} \psi_{a}$ and $\varphi(t)$ is a real function depending functionally on $E_{a}$ and $\psi_{a}$.

- Evolution of expectation value of an observable $B$ given by

$$
\langle B\rangle_{t}=\left(\psi_{a(t)}, B \psi_{a(t)}\right) .
$$

\section{Backreaction - determination of $a(t)$}

- Intrinsic energy stored in part $Q$ of the system represented by $H$, which in the coupled system is not a constant of motion any more; its expectation value

$$
\mathcal{E}_{a}:=\left(\psi_{a}, H \psi_{a}\right)
$$

depends on time through variables $a$.

- Changes in $\mathcal{E}_{a}$ correspond to the energy which has been transferred from $Q$ to the rest of the system, which is described by the variables $a$ (effectively - with the suppression of all microscopic details of $M$ ). Thus $\mathcal{E}_{a}$ plays the role of a potential energy with respect to these variables. We assume that the rest of the total energy of the coupled system is supplied by the kinetic energy of $M$, thus we obtain a potential system, with the generalized force given by

$$
\mathcal{F}_{a}=-\frac{\partial \mathcal{E}_{a}}{\partial a}
$$

\section{A class of quasi-free systems}

\section{Classical system}

- Symplectic space (phase space): $\mathcal{L} \subset \mathcal{R} \oplus \mathcal{R} \ni V \equiv(v \oplus u), \quad \mathcal{R}$ - real Hilbert space; symplectic form

$$
\sigma\left(V_{1}, V_{2}\right)=\left(v_{2}, u_{1}\right)-\left(v_{1}, u_{2}\right) .
$$

- Hamiltonian function

$$
\mathcal{H}(v, u)=\frac{1}{2}[(u, u)+(h v, h v)],
$$

$h$ - positive selfadjoint operator on $\mathcal{R}$.

- Symplectic evolution

$$
T_{t}(v \oplus u)=\left(\cos (h t) v+\sin (h t) h^{-1} u\right) \oplus(-\sin (h t) h v+\cos (h t) u) .
$$


- Denote

$$
V^{\prime}(V)=\left(v^{\prime}, u\right)+\left(u^{\prime}, v\right)
$$

then

$$
\left(T_{t} V^{\prime}\right)(V)=V^{\prime}\left(T_{t} V\right)
$$

\section{Quantum system}

- 'Quantization': $V^{\prime}(V) \rightarrow \Phi(V)$ - algebraic elements satisfying CCR

$$
\left[\Phi\left(V_{1}\right), \Phi\left(V_{2}\right)\right]=i \sigma\left(V_{1}, V_{2}\right) \text { id }, \quad V \in \mathcal{L},
$$

evolution

$$
\alpha_{t}(\Phi(V))=\Phi\left(T_{t} V\right) .
$$

- Vacuum representation $\Phi(V) \rightarrow \Phi_{0}(V)$ - operators in a Fock space; representation defined by demands:

$$
\Phi_{0}\left(T_{t} V\right)=U(t) \Phi_{0}(V) U^{*}(t), \quad U(t)=\exp (i t H)
$$

$H$ - positive, with ground state vector $\Omega$.

\section{Examples}

- Free massless scalar field - initial value formulation:

$$
\mathcal{R}=L_{\mathbb{R}}^{2}\left(\mathbb{R}^{3}\right), \quad h=\sqrt{-\Delta}, \quad \mathcal{L}=\mathcal{D}_{\mathbb{R}}\left(\mathbb{R}^{3}\right) \oplus \mathcal{D}_{\mathbb{R}}\left(\mathbb{R}^{3}\right) .
$$

- Scalar field with boundary conditions on surfaces coordinated by parameters $a$ :

$$
\mathcal{R}=L_{\mathbb{R}}^{2}\left(\mathbb{R}^{3}\right),\left[h_{a}^{B}\right]^{2}=-\Delta_{\text {b.c. }}, \mathcal{L}_{a}^{B}=\mathcal{D}_{\mathcal{R}}\left(h_{a}^{B}\right) \oplus \mathcal{D}_{\mathcal{R}}\left(\left[h_{a}^{B}\right]^{-1 / 2}\right) .
$$

- 'Momentum'-regularized boundary conditions: $h_{a}=f\left(h, h_{a}^{B}\right)$, such that $h_{a} \simeq h_{a}^{B}$ for small momentum transfer, and $h_{a} \simeq h$ for large momentum transfer (to be made more precise in specific models).

- Scalar field with external static interaction depending on parameters $a$ : $h_{a}^{2}=-\Delta+V_{a}, V_{a}$ - perturbation .

\section{Examples - quantization}

- Algebra CCR

$$
\left[\Phi\left(V_{1}\right), \Phi\left(V_{2}\right)\right]=i \sigma\left(V_{1}, V_{2}\right) \mathrm{id}, \quad V \in \mathcal{L}_{a}
$$

evolution

$$
\alpha_{a t}(\Phi(V))=\Phi\left(T_{a t} V\right),
$$

where $T_{a t}$ is defined by $h_{a}$. 
- Ground state representation $\Phi(V) \rightarrow \Phi_{a}(V)$ - operators in a Fock space; representation defined by demands:

$$
\Phi_{a}\left(T_{t} V\right)=U_{a}(t) \Phi_{a}(V) U_{a}^{*}(t), \quad U_{a}(t)=\exp \left(i t H_{a}\right),
$$

$H_{a}$-positive, with ground state vector $\Omega_{a}$.

\section{Conditions for admissibility of models}

- Stability of algebras: $\quad \mathcal{L}=\mathcal{L}_{a}$ (as a CCR algebra is uniquely determined by the symplectic space over which it is build).

Not satisfied in the sharp boundary conditions case!

(And no way to satisfy the condition by any extension of symplectic spaces.)

- Relation between representations: when stability of algebras is ensured then annihilation/creation operators of representations determined by $h$ and $h_{a}$ are related by a Bogoljubov transformation

$$
a_{a}(f)=a\left(T_{a} f\right)+a^{*}\left(S_{a} f\right), a_{a}^{*}(f)=a^{*}\left(T_{a} f\right)+a\left(S_{a} f\right),
$$

with $T_{a}$ linear and $S_{a}$ antilinear, determined by $h$ and $h_{a}$.

- Representations are equivalent iff

$$
\mathcal{N}_{a} \equiv \operatorname{Tr}\left[S_{a} S_{a}^{*}\right]=\frac{1}{4} \operatorname{Tr}\left[h^{-1 / 2}\left(h_{a}-h\right) h_{a}^{-1}\left(h_{a}-h\right) h^{-1 / 2}\right]<\infty .
$$

Then $\mathcal{N}_{a}=\left(\Omega_{a}, N \Omega_{a}\right), N$ - particle (or 'excitation') number.

- Casimir energy for ground state

$$
\mathcal{E}_{a}=\left(\Omega_{a}, H \Omega_{a}\right)=\frac{1}{4} \operatorname{Tr}\left[\left(h_{a}-h\right) h_{a}^{-1}\left(h_{a}-h\right)\right] .
$$

If this happened to be infinite, this would have a perfectly legitimate physical meaning: creation of $\Omega_{a}$, although theoretically possible, needs infinite amount of energy.

\section{Two models for parallel planes}

\section{Planar symmetry}

- $\mathcal{R}=L_{\mathbb{R}}^{2}\left(\mathbb{R}^{2}\right) \otimes L_{\mathbb{R}}^{2}(\mathbb{R})$,

$$
\begin{gathered}
h^{2}=\left(h_{\perp} \otimes \mathrm{id}\right)^{2}+\left(\mathrm{id} \otimes h_{z}\right)^{2}, \quad h_{a}^{2}=\left(h_{\perp} \otimes \mathrm{id}\right)^{2}+\left(\mathrm{id} \otimes h_{z a}\right)^{2} \\
h_{\perp}^{2}=-\Delta_{\perp}, \quad h_{z}^{2}=-\partial_{z}^{2}
\end{gathered}
$$

- $h_{z a}$ will model parallel planes at a distance $a$.

- $\mathcal{N}_{a}$ and $\mathcal{E}_{a}$ must be normalized to quantities per unit area of planes, $n_{a}$ and $\varepsilon_{a}$ resp.

\section{Two models}

- (i) A model with transparency to large momenta:

$$
h_{z a}=h_{z}+G\left(h_{z}\right)\left[F\left(h_{z a}^{B}\right)-F\left(h_{z}\right)\right] G\left(h_{z}\right),
$$

$h_{z a}^{B}$ - sharp Dirichlet or Neumann boundary conditions at points separated by $a$, $F, G(p) \rightarrow 0(p \rightarrow \infty), \quad F(p)=p, G(p)=1\left(p \leq p_{0}\right)$. 
- (ii) A model with nonlocality control:

$$
h_{z a}^{2}=h_{z}^{2}+V_{a}, \quad V_{a}\left(z, z^{\prime}\right)=g(z-b) \overline{g\left(z^{\prime}-b\right)}+g(z+b) \overline{g\left(z^{\prime}+b\right)}
$$

$g$ of compact support, even $(D)$ or odd $(N)(b=a / 2)$.

- Both classes of models meet the admissibility criteria, so the Casimir energy per area $\varepsilon_{a}$ is well defined and finite for all $a$.

\section{Scaling}

\section{Approximating boundary conditions}

- For each model - a one parameter $(\lambda \in(0,1\rangle)$ family of scaled models, such that for fixed $a$ and $\lambda \rightarrow 0$ the sharp boundary conditions are recovered.

- The Casimir energy per area scales:

$$
\varepsilon_{a, \lambda}=\lambda^{-3} \varepsilon_{a / \lambda} .
$$

Thus to find scaling behavior of $\varepsilon_{a, \lambda}$ for scaled models: expand $\varepsilon_{a}$ in powers of $1 / a$ up to third order.

\section{Expanding $\varepsilon_{a}$}

- Casimir energy $\varepsilon_{a}$ given by a complex integral expression depending functionally on functions defining the models and on $a$.

- Expansion:

$$
\varepsilon_{a}=\varepsilon_{\infty}+\frac{\gamma}{a^{2}}-\frac{\pi^{2}}{1440 a^{3}}+\text { higher terms },
$$

$\varepsilon_{\infty}-$ energy needed to produce field configuration around two independent plates $\gamma-\quad=0$ for Dirichlet case, and model dependent for Neumann case - contribution from inside the walls, $a^{-3}$-term - universal for large class of models.

- Models for conducting plates and electromagnetic field - sum of the D and N terms.

\section{Summary and outlook}

- Proper formulation of the problem removes the usual sources of infinities.

- Casimir energy defined as expectation value of one and the same observable for all modifications of external conditions in question.

- Models considered very simplified, but showing the strength of the formulation.

- Large area for further research: more refined models of external bodies, thermal states, different geometries,...

\section{References}

1. R. Haag, Local Quantum Physics, 2nd edn. (Springer, Berlin, 1996).

2. A. Herdegen, No nonsense Casimir force, Acta Phys.Pol. B 32 (2001) 55-63. 
3. A.Herdegen, Quantum backreaction (Casimir) effect. I. What are admissible idealizations?, Ann. Henri Poncaré 6 (2005) 657-695.

4. A.Herdegen, Quantum backreaction (Casimir) effect. II. Scalar and electromagnetic fields, Ann. Henri Poncaré 7 (2006) 253-301.

5. A. Herdegen and M. Stopa, Global versus local Casimir effect, Ann. Henri Poncaré 11 (2010) 1171-1200. 